\title{
Novel Rogue Waves for a Mixed Coupled Nonlinear Schrödinger Equation on Darboux-Dressing Transformation
}

\author{
Min-Jie Dong ${ }^{1}$, Li-Xin Tian ${ }^{1,2, *}$ and Jing-Dong $\mathrm{Wei}^{2}$ \\ ${ }^{1}$ School of Mathematical Sciences, Nanjing Normal University, Nanjing, \\ Jiangsu 210046, P.R. China. \\ ${ }^{2}$ School of Mathematical Sciences, Jiangsu University, Zhenjiang, Jiangsu \\ 212013, P.R. China. \\ Received 18 November 2020; Accepted (in revised version) 31 May 2021.
}

\begin{abstract}
Focusing-defocusing mixed coupled nonlinear Schrödinger equation of localised waves in a two-mode nonlinear fiber is investigated. Novel localised wave solutions are constructed by employing the Darboux-dressing transformation. The set of such solutions includes rogue waves on the soliton background. In addition, for the main characteristics of these solutions, we give the graphs to make readers more aware of the characteristics of these solutions. Hopefully our results can be used to help enrich rogue waves phenomena in nonlinear wave field.
\end{abstract}

AMS subject classifications: 65M10, 78A48

Key words: Mixed coupled nonlinear Schrödinger equation, Darboux-dressing transformation, breather wave, rogue wave.

\section{Introduction}

Rogue waves are used to describe huge catastrophic waves unexpectedly arising on relatively calm ocean surface [12] and to characterise extreme wave events in optics [20], plasma [16], Bose-Einstein condensate [5], finance [29,30], and so on. It is the common belief that rogue waves have three main characteristics:

1) The amplitude of the wave is more than twice (or larger) than the average amplitude of the significant wave height [1].

2) They appear from nowhere and disappear without trace [2].

3) The probability distribution function of the amplitude obeys the unusual $L$-shaped statistics, which means that the frequency of the wave is higher than predicted by the classical Gaussian distribution $[1,20]$.

${ }^{*}$ Corresponding author. Email addresses: dongminj iepoppy@126.com (M.-J. Dong), tianlx@uj s.edu .cn (L.-X. Tian), weijingdong@ujs.edu.cn (J.-D. Wei) 
Recently, rogue waves have been encountered in optical fibers, deep water waves and other fields where nonlinear Schrödinger (NLS) equations are employed. In particular, Peregrine [19] constructed a first-order rational solution of the NLS equation by mathematical method, which is a formal description of a single rogue wave. This solution, the peak amplitude of which is three times of the average height, was later named after him. The rogue waves attracted a great attention in recent years - cf. [6, 7, 10,31,34,35], and it is worth noting their close connection to NLS and coupled nonlinear Schrödinger equation(CNLS), Li et al. [13] determined reduced and non-reduced vector rogue wave solutions of CNLS using the generalised Darboux transformation (DT), Feng et al. [8] employed DT in order to construct multi-breather solutions of NLS on the background of elliptic functions and expressed them via theta functions, Zhang et al. [33] used DT in new localised wave solutions; and so on.

On the other hand, the Darboux-dressing transformation has been used in the study of the classical Schrödinger equation [17], integrable vector nonlinear Schrödinger equations [18], the Manakov system [23], the Kundu-nonlinear Schrödinger equation [25], the coupled cubic-quintic nonlinear Schrödinger equations [28] and in other fields [24,26, 27,32].

Numerous works are devoted the two-component case (as so called the Manakov system)

$$
\begin{aligned}
& i u_{t}+\frac{1}{2} u_{x x}+\sigma\left(|u|^{2}+|v|^{2}\right) u=0 \\
& i v_{t}+\frac{1}{2} v_{x x}+\sigma\left(|u|^{2}+|v|^{2}\right) v=0
\end{aligned}
$$

where $u(x, t)$ and $v(x, t)$ are wave envelopes, and $x$ and $t$ are, respectively, transverse and longitudinal coordinates [3,9]. Every subscripted variable in the Eqs. (1.1) refers to the partial differentiation. If $\sigma=1$, the equations represent the defocusing case, and if $\sigma \neq 1$ the focusing case.

In this work, we consider breather and rogue waves of the focusing-defocusing mixed coupled nonlinear Schrödinger equation (mCNLSEs)

$$
\begin{aligned}
& i u_{t}+\frac{1}{2} u_{x x}+\left(|v|^{2}-|u|^{2}\right) u=0 \\
& i v_{t}+\frac{1}{2} v_{x x}+\left(|v|^{2}-|u|^{2}\right) v=0
\end{aligned}
$$

where $u$ and $v$ are respectively related to the focusing and defocusing type nonlinearities [21]. The terms $|u|^{2} u,|v|^{2} v$ and $|v|^{2} u,|u|^{2} v$ are self-phase and cross-phase modulations. The Eqs. (1.2) can be regarded as a mixture of the defocusing and focusing conditions of the Manakov system (1.1) and are vigorously studied. For example, Tian et al. [21] considered initial-boundary value problems related to the Fokas method, Vijayajayanthi et al. [22] studied bright-dark solitons and their collisions in mixed $N$-coupled nonlinear Schrödinger equations, Kanna et al. [11] investigated the soliton collisions with a shape change by intensity redistribution, Ling et al. [14] constructed vector rogue wave and bright-dark rogue wave solutions by using the Darboux transformation. However, to the best of the our knowl- 
edge, rogue wave and breather wave solutions are still not connected to the Darboux dressing transformation [17].

This article is organised as follows. In Section 2, we follows the considerations [14,21] and determine the Lax pair for the Eqs. (1.2). This Lax pair in used in the construction of the corresponding Darboux transformation and an asymptotic expansion. In Section 3, we construct exact breather wave solutions. In Section 4, we give the higher order rogue wave solutions related to the Darboux-dressing transformation and Taylor series expansion. We visualise certain solutions to discuss interesting nonlinear phenomena. Finally, our conclusions are given in Section 5.

\section{Darboux-Dressing Transformation}

Because of complete integrability [14,21], the Lax pair of the Eqs. (1.2) appears as the compatibility condition $\Phi_{t x}=\Phi_{x t}$ for the following pair of linear equations:

$$
\Phi_{x}=U \Phi, \quad \Phi_{t}=V \Phi,
$$

where $\Phi=\left(\phi_{1}, \phi_{2}, \phi_{3}\right)^{T}$ is a vector eigenfunction in $\mathbb{C}^{2}, \phi_{1}, \phi_{2}$ and $\phi_{3}$ are the complex functions of variables $x$ and $t, T$ is the operation of matrix transposition. Besides, $U, V$ are the $3 \times 3$ square matrices,

$$
U=\left(\begin{array}{ccc}
i \lambda & -i u^{*} & i v^{*} \\
i u & -i \lambda & 0 \\
i v & 0 & -i \lambda
\end{array}\right), \quad V=\left(\begin{array}{ccc}
i \lambda^{2}+\frac{1}{2} i\left(u^{*} u-v^{*} v\right) & -i \lambda u^{*}-\frac{1}{2} u_{x}^{*} & i \lambda v^{*}+\frac{1}{2} v_{x}^{*} \\
i \lambda u-\frac{1}{2} u_{x} & -i \lambda^{2}-\frac{1}{2} i u^{*} u & \frac{1}{2} i u v^{*} \\
i \lambda v-\frac{1}{2} v_{x} & -\frac{1}{2} i v u^{*} & -i \lambda^{2}+\frac{1}{2} i v^{*} v
\end{array}\right)
$$

where $\lambda \in \mathbb{C}$ is the spectral parameter and $u^{*}$ and $v^{*}$ are the complex conjugate of $u$ and $v$, respectively.

Based on the study of $[14,21]$, an appropriate Darboux transformation for the Eqs. (1.2) can be constructed as follows.

Theorem 2.1. The N-folds Darboux transformation has the form

$$
\Phi_{[N]}=\nabla \Phi, \quad \nabla=I_{3}-\frac{\left(\lambda_{1}-\lambda_{1}^{*}\right)}{\left(\lambda-\lambda_{1}^{*}\right)} \frac{\Lambda_{[N-1]} \Lambda_{[N-1]}^{*}}{\Lambda_{[N-1]}^{*} \Lambda_{[N-1]}},
$$

where

$$
\left(\begin{array}{c}
u_{[N]} \\
v_{[N]}
\end{array}\right)=\left(\begin{array}{c}
u_{[N-1]} \\
v_{[N-1]}
\end{array}\right)+\frac{2\left(\lambda_{1}^{*}-\lambda_{1}\right) \phi_{[1 N-1]}^{*}}{\left|\phi_{1[N-1]}\right|^{2}+\left|\phi_{2[N-1]}\right|^{2}+\left|\phi_{3[N-1]}\right|^{2}} \times\left(\begin{array}{c}
\phi_{2[N-1]} \\
\phi_{3[N-1]}
\end{array}\right),
$$

and

$$
I_{3}=\operatorname{diag}(1,1,1), \quad \Lambda_{[N-1]}=\Phi\left(x, t, \lambda_{1}\right) Z_{[N-1]}=\left(\phi_{1[N-1]}, \phi_{2[N-1]}, \phi_{3[N-1]}\right)^{T}
$$

with

$$
Z_{[N-1]}=\left(z_{1[N-1]}, z_{[2 N-1]}, z_{3[N-1]}\right)^{T}
$$


being column vector contained free real parameters, $\Phi$ is the fundamental solution of the Lax equation (2.1) with $\lambda=\lambda_{1}$ depending on the variables $x$ and $t$, $*$ denotes the conjugate, $\Phi$ is a column vector function of $\lambda$.

Proof. The proof is similar to the proofs in $[14,17,27]$ and is omitted here.

We discover that Ref. [14] mainly uses matrix analysis method to solve mCNLS equations and to obtain complete classification of non-singular solutions. In this paper, the breather solution is obtained based on the DT equation and the seed solution, and the rogue wave is obtained by using Taylor expansion. The main differences are:

1. Kuznetsov-Ma soliton and Akhmediev breather are verified.

2. By changing the parameters, the Peregrine structure and the boomeron type bright soliton are obtained.

3. The second-order rogue wave are constructed in this paper.

\section{Breather Wave Solutions}

To derive the exact breather wave solutions of the Eqs. (1.2), we start with seed solutions of the form

$$
u=a_{1} \exp (i k x+i w t), \quad v=a_{2} \exp (i k x+i w t)
$$

with the dispersion relations

$$
w=-a_{1}^{2}+a_{2}^{2}-\frac{1}{2} k^{2},
$$

where $a_{1}$ and $a_{2}$ are real parameters and $k$ is the wave number. According to [17, 18, 27], the corresponding solutions of the Lax system (2.1) have the form

$$
\begin{aligned}
& \Phi=\left(\begin{array}{l}
\phi_{1}(x, t) \\
\phi_{2}(x, t) \\
\phi_{3}(x, t)
\end{array}\right)=A F G Z, \\
& F=e^{i \Theta x}, \quad G=e^{i \Lambda t}
\end{aligned}
$$

where

$$
A=\left(\begin{array}{ccc}
1 & 0 & 0 \\
0 & \exp (i k x+i w t) & 0 \\
0 & 0 & \exp (i k x+i w t)
\end{array}\right),
$$

and $Z=\left(z_{1}, z_{2}, z_{3}\right)^{\top}$ is a free complex vector.

Substituting $\Phi$ (3.2) into the Lax pairs (2.1), leads to the following representations of $\Theta$ and $\Lambda$ :

$$
\Theta=\left(\begin{array}{ccc}
\lambda & -a_{1} & a_{2} \\
a_{1} & -k-\lambda & 0 \\
a_{2} & 0 & -k-\lambda
\end{array}\right)
$$




$$
\Lambda=\left(\begin{array}{ccc}
\lambda^{2}+\frac{1}{2} a_{1}^{2}-\frac{1}{2} a_{2}^{2} & \frac{1}{2} a_{1}(-2 \lambda+k) & -\frac{1}{2} a_{2}(-2 \lambda+k) \\
-\frac{1}{2} a_{1}(-2 \lambda+k) & -\lambda^{2}-\frac{1}{2} a_{1}^{2}-w & \frac{1}{2} a_{1} a_{2} \\
-\frac{1}{2} a_{1}(-2 \lambda+k) & -\frac{1}{2} a_{1} a_{2} & -\lambda^{2}+\frac{1}{2} a_{2}^{2}-w
\end{array}\right)
$$

It is easily seen that (3.4) satisfies

$$
\begin{aligned}
& {[\Theta, \Lambda]=\Theta \Lambda-\Lambda \Theta=0,} \\
& A_{x}+i A \Theta-U A=0, \\
& A_{t}+i A \Lambda-V A=0
\end{aligned}
$$

According to the Eq. (3.2), the matrix $F$ can be written as

$$
F=\exp \left(-\frac{i k x}{2}\right)\left(\begin{array}{ccc}
f_{11} & -2 i a_{1} \tau \sinh \left(\frac{1}{2} \tau x\right) & 2 i a_{2} \tau \sinh \left(\frac{1}{2} \tau x\right) \\
2 i a_{1} \tau \sinh \left(\frac{1}{2} \tau x\right) & f_{22} & f_{23} \\
2 i a_{2} \tau \sinh \left(\frac{1}{2} \tau x\right) & f_{32} & f_{33}
\end{array}\right),
$$

where

$$
\begin{aligned}
& \tau=\sqrt{4\left(a_{1}^{2}-a_{2}^{2}\right)-k^{2}-4 k \lambda-4 \lambda^{2}} \\
& f_{11}=i \tau(k+2 \lambda) \sinh \left(\frac{1}{2} \tau x\right)+\tau^{2} \cosh \left(\frac{1}{2} \tau x\right) \\
& f_{22}=-\frac{\tau}{a_{1}^{2}-a_{2}^{2}}\left((i k+2 i \lambda) a_{1}^{2} \sinh \left(\frac{1}{2} \tau x\right)-a_{1}^{2} \tau \cosh \left(\frac{1}{2} \tau x\right)+a_{2}^{2} \tau \exp \left(-\frac{1}{2} i x(k+2 \lambda)\right)\right), \\
& f_{23}=\frac{a_{1} a_{2} \tau}{a_{1}^{2}-a_{2}^{2}}\left((i k+2 i \lambda) \sinh \left(\frac{1}{2} \tau x\right)-\tau \cosh \left(\frac{1}{2} \tau x\right)+\tau \exp \left(-\frac{1}{2} i x(k+2 \lambda)\right)\right) \\
& f_{32}=-\frac{a_{1} a_{2} \tau}{a_{1}^{2}-a_{2}^{2}}\left((i k+2 i \lambda) \sinh \left(\frac{1}{2} \tau x\right)-\tau \cosh \left(\frac{1}{2} \tau x\right)+\tau \exp \left(-\frac{1}{2} i x(k+2 \lambda)\right)\right), \\
& f_{33}=\frac{\tau}{a_{1}^{2}-a_{2}^{2}}\left((i k+2 i \lambda) a_{2}^{2} \sinh \left(\frac{1}{2} \tau x\right)-a_{2}^{2} \tau \cosh \left(\frac{1}{2} \tau x\right)+a_{1}^{2} \tau \exp \left(-\frac{1}{2} i x(k+2 \lambda)\right)\right) .
\end{aligned}
$$

Similar calculations yield

$$
G=\exp \left(-\frac{i w t}{2}\right)\left(\begin{array}{lll}
g_{11} & g_{12} & g_{13} \\
g_{21} & g_{22} & g_{23} \\
g_{31} & g_{32} & g_{33}
\end{array}\right)
$$

where

$$
\begin{aligned}
& v=\sqrt{\left(a_{1}^{2}-a_{2}^{2}\right) k^{2}+4 \lambda\left(a_{1}^{2}-a_{2}^{2}\right) k-w^{2}+\left(-2 a_{1}^{2}+2 a_{2}^{2}-4 \lambda^{2}\right) w-a_{2}^{4}+2 a_{1}^{2} a_{2}^{2}-a_{2}^{4}-4 \lambda^{4}}, \\
& g_{11}=i\left(a_{1}^{2}-a_{2}^{2}+2 \lambda^{2}+w\right) v \sinh \left(\frac{1}{2} v t\right)+v^{2} \cosh \left(\frac{1}{2} v t\right),
\end{aligned}
$$




$$
\begin{aligned}
& g_{12}=i a_{1} v(k-2 \lambda) \sinh \left(\frac{1}{2} v t\right), \quad g_{13}=-i a_{2} v(k-2 \lambda) \sinh \left(\frac{1}{2} v t\right), \\
& g_{22}=-\frac{v}{a_{1}^{2}-a_{2}^{2}}\left(i\left(a_{1}^{4}-a_{1}^{2} a_{2}^{2}+2 a_{1}^{2} \lambda^{2}+a_{1}^{2} w\right) \sinh \left(\frac{1}{2} v t\right)-a_{1}^{2} v \cosh \left(\frac{1}{2} v t\right)\right. \\
& \left.+a_{2}^{2} v \exp \left(-\frac{1}{2} i t\left(2 \lambda^{2}+w\right)\right)\right) \\
& g_{23}=\frac{a_{1} a_{2} \tau}{a_{1}^{2}-a_{2}^{2}}\left(i\left(a_{1}^{2}-a_{2}^{2}+2 \lambda^{2}+w\right) \sinh \left(\frac{1}{2} v t\right)-v \cosh \left(\frac{1}{2} v t\right)\right. \\
& \left.+v \exp \left(-\frac{1}{2} i t\left(2 \lambda^{2}+w\right)\right)\right) \\
& g_{32}=-\frac{a_{1} a_{2} \tau}{a_{1}^{2}-a_{2}^{2}}\left(i\left(a_{1}^{2}-a_{2}^{2}+2 \lambda^{2}+w\right) \sinh \left(\frac{1}{2} v t\right)-v \cosh \left(\frac{1}{2} v t\right)\right. \\
& \left.+v \exp \left(-\frac{1}{2} i t\left(2 \lambda^{2}+w\right)\right)\right) \\
& g_{33}=\frac{v}{a_{1}^{2}-a_{2}^{2}}\left(i\left(a_{1}^{2} a_{2}^{2}-a_{2}^{4}+2 a_{2}^{2} \lambda^{2}+a_{2}^{2} w\right) \sinh \left(\frac{1}{2} v t\right)-a_{2}^{2} v \cosh \left(\frac{1}{2} v t\right)\right. \\
& \left.+a_{1}^{2} v \exp \left(-\frac{1}{2} i t\left(2 \lambda^{2}+w\right)\right)\right) \\
& g_{12}=-g_{21}, \quad g_{13}=g_{31} .
\end{aligned}
$$

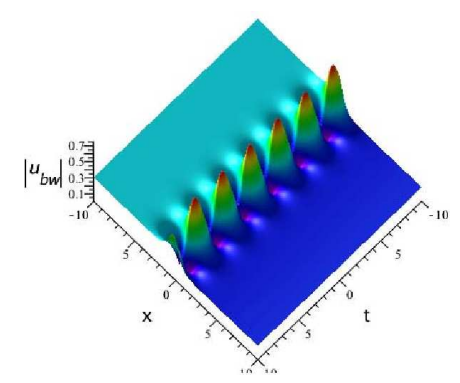

a)

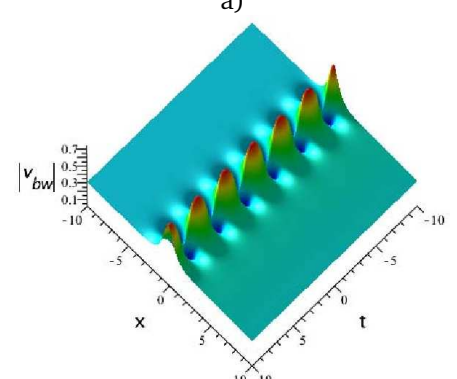

d)

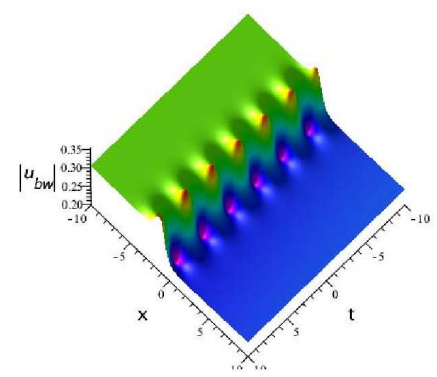

b)

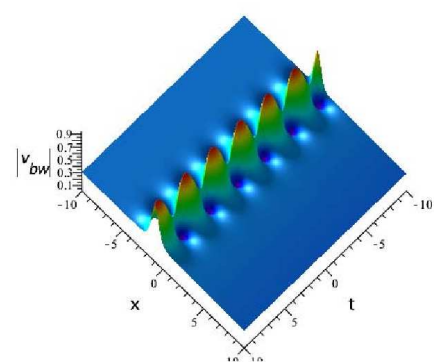

e)

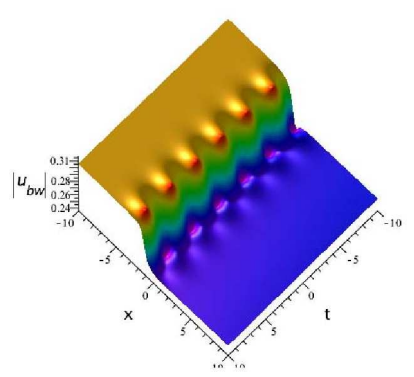

c)

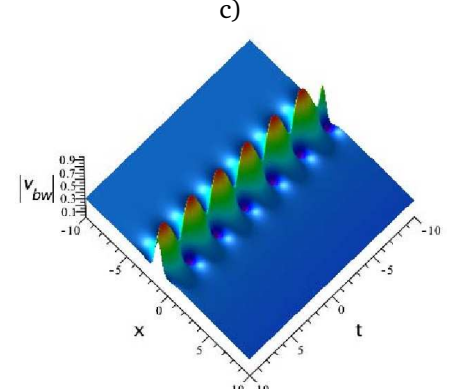

f)

Figure 1: Akhmediev breathers, $a_{1}=i / 4, a_{2}=1 / 4, k=0, \lambda=i, z_{1}=1, z_{2}=1$. $(a, d) z_{3}=1,(b, e) z_{3}=10$, $(c, f) z_{3}=10000$. 


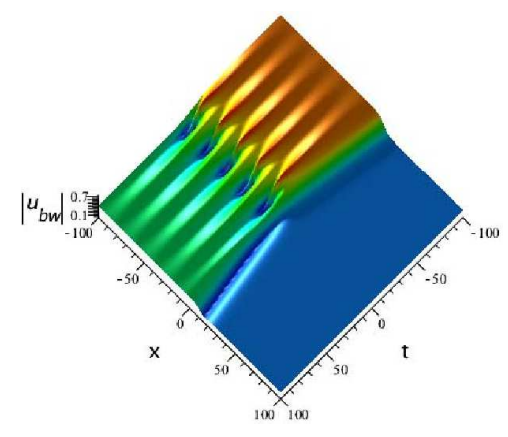

a)

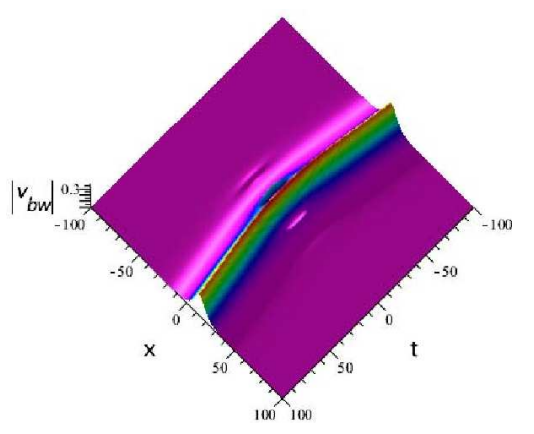

b)

Figure 2: Kuznetsov-Ma solutions, $a_{1}=i / 4, a_{2}=0, k=0, \lambda=1 / 5 i, z_{1}=1, z_{2}=1, z_{3}=1$.

Substituting (3.5) and (3.6) into (3.1), and according to Theorem 2.1, the corresponding novel breathers are displayed in Figs. 1 and 2.

Solutions in Fig. 1 demonstrate time locality and space periodicity, which is called Akhmediev breathers [4]. Fig. 2 has opposite characteristics, which is called KuznetsovMa soliton [15].

\section{Novel Rogue Waves}

To construct novel rogue wave solutions of the Eqs. (1.2) of exponential and polynomial functions via Taylor expansions formulas, we choose $\lambda= \pm i \theta(1+\varepsilon), 0<\varepsilon<1, \theta=$ $\sqrt{-a_{1}^{2}+a_{2}^{2}}$. The corresponding breather wave solutions (3.2),(3.3) have the form

$$
\begin{aligned}
& \left.F(\lambda)\right|_{\lambda= \pm i \theta(1+\varepsilon)}=\Sigma_{N=0}^{\infty} F_{N} \varepsilon^{N} \\
& \left.G(\lambda)\right|_{\lambda= \pm i \theta(1+\varepsilon)}=\exp \left(\frac{\theta^{2} i t}{2}\right) \Sigma_{N=0}^{\infty} G_{N} \varepsilon^{N}
\end{aligned}
$$

where $F_{N}, G_{N}$ are the $N$-th coefficient matrices of $\varepsilon$. Analogously,

$$
Z_{\varsigma}=\Sigma_{\varsigma=0}^{N}\left(z_{1 \varsigma}, z_{2 \varsigma}, z_{3 \varsigma}\right) \varepsilon^{\varsigma}
$$

and

$$
\begin{aligned}
& \Phi(\lambda)=\left.\right|_{\lambda= \pm i \theta(1+\varepsilon)}=\Sigma_{N=0}^{\infty} \Phi_{N} \varepsilon^{N}, \\
& \Phi_{N}=\mho_{0}=\exp \left(\frac{\theta^{2} i t}{2}\right) A \Sigma_{\varsigma=0}^{N} \Sigma_{\iota}^{N} F_{\varsigma} G_{\iota}\left(z_{1, N-\varsigma-\iota}, z_{2 \varsigma, N-\varsigma-\iota}, z_{3 \zeta, N-\varsigma-\iota}\right) .
\end{aligned}
$$

Moreover, novel rogue waves $\Sigma_{\varsigma=0}^{\infty}\left(z_{1 \varsigma}, z_{2 \varsigma}, z_{3 \varsigma}\right) \varepsilon^{\varsigma}$ can be written as

$$
\Sigma_{\varsigma=0}^{\infty}\left(z_{1 \varsigma}, z_{2 \varsigma}, z_{3 \varsigma}\right) \varepsilon^{\varsigma}=\left.\exp (i \Theta X+i \Lambda T) L\right|_{\lambda= \pm i \theta(1+\varepsilon)},
$$

where

$$
X=\Sigma_{\zeta=0}^{N} R_{N} \varepsilon^{N}, \quad T=\Sigma_{\varsigma=0}^{N} S_{N} \varepsilon^{N}, \quad L=\left(L_{1}, L_{2}, L_{3}\right)^{\prime} .
$$


We can use Theorem 2.1 to obtain new higher order rogue waves. For $N=1$, the first-order rogue waves have the form

$$
\left(\begin{array}{c}
u_{[r 1]} \\
v_{[r 1]}
\end{array}\right)=\left(\begin{array}{c}
u_{[0]} \\
v_{[0]}
\end{array}\right)+\frac{2\left(\lambda_{1}^{*}-\lambda_{1}\right) \phi_{1[0]}^{*}}{\left|\phi_{1[0]}\right|^{2}+\left|\phi_{2[0]}\right|^{2}+\left|\phi_{3[0]}\right|^{2}} \times\left(\begin{array}{c}
\phi_{2[0]} \\
\phi_{3[0]}
\end{array}\right),
$$

where

$$
\begin{aligned}
& \left(\begin{array}{l}
\varphi_{1[0]} \\
\varphi_{2[0]} \\
\varphi_{3[0]}
\end{array}\right)=\mho_{0}=\exp \left(\frac{\theta^{2} i t}{2}\right) A F_{0} G_{0} Z_{0}, \quad Z_{0}=\left(\begin{array}{c}
z_{1[0]} \\
z_{2[0]} \\
z_{3[0]}
\end{array}\right), \\
& F_{0}=\left(\begin{array}{ccc}
1-\theta x & -i a_{1} x & i a_{2} x \\
i a_{1} x & \frac{-\theta a_{1}^{2} x+\exp (\theta x) a_{2}^{2}-a_{1}^{2}}{\theta^{2}} & \frac{a_{1} a_{2}(\theta x-\exp (\theta x)+1)}{\theta^{2}} \\
i a_{2} x & \frac{a_{1} a_{2}(-\theta x+\exp (\theta x)-1)}{\theta^{2}} & \frac{-\theta a_{2}^{2} x+\exp (\theta x) a_{1}^{2}-a_{2}^{2}}{\theta^{2}}
\end{array}\right), \\
& G_{0}=\left(\begin{array}{ccc}
1-i t \theta^{2} & a_{1} t \theta & \frac{a_{1} a_{2}}{\theta^{2}}\left(i t \theta^{2}+1-\exp \left(\frac{\theta^{2} i t}{2}\right)\right) \\
-a_{1} t \theta & \frac{1}{\theta^{2}}\left(\chi_{1}\right) & \frac{1}{\theta^{2}}\left(\chi_{2}\right) \\
-a_{2} t \theta & \frac{a_{1} a_{2}}{\theta^{2}}\left(i t \theta^{2}+1-\exp \left(\frac{\theta^{2} i t}{2}\right)\right) & \chi_{2}=i a_{2}^{4} t-i a_{1}^{2} a_{2}^{2} t+a_{2}^{2}-a_{1}^{2} \exp \left(\frac{\theta^{2} i t}{2}\right) .
\end{array}\right.
\end{aligned}
$$

Fig. 3 shows three different types of first-order rogue waves to illustrate rogue waves (4.1).

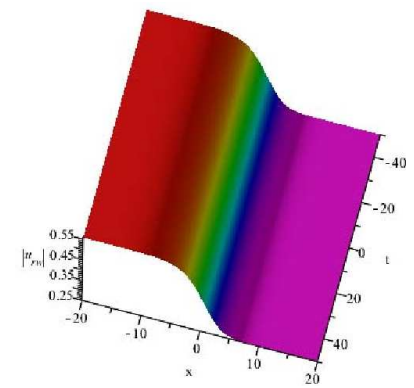

a)

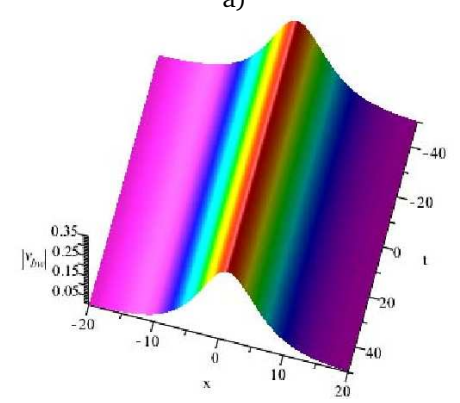

d)

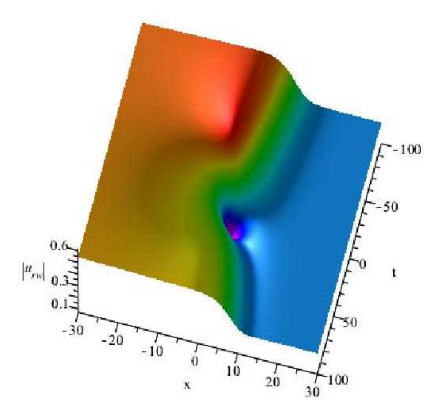

b)

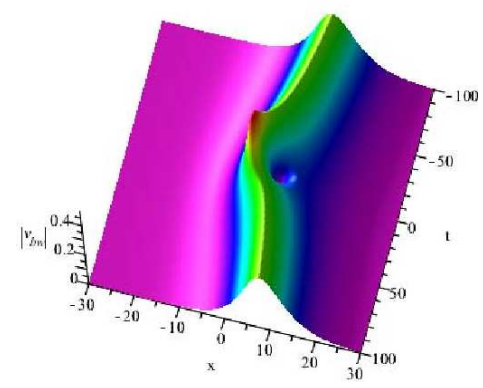

e)

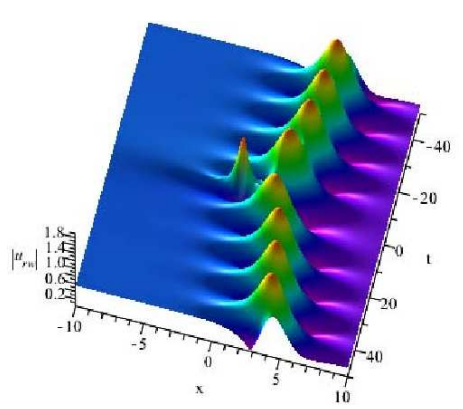

c)

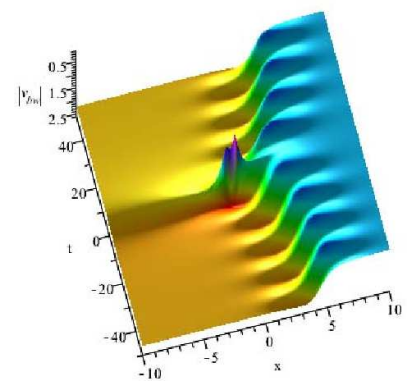

f)

Figure 3: First-order rogue waves from Eqs. (4.1), $a_{1}=i / 4, a_{2}=0, k=0, \lambda=1 / 5 i, z_{1}=1, z_{2}=1$. $(a, d)$ $z_{3}=1,(b, e) z_{3}=10,(c, f) z_{3}=10000$. 
Figs. 3(a,b,d,e) show that the Peregrine structure and boomeron type bright solitons appear with the increase of $z_{3}$. According to Figs. 3(b,e), the first-order rogue waves interact with an amplitude-varying soliton in $u$ and one bright soliton and the first-order rogue waves in $v$. The Peregrine bump coexists and interacts with breather-like solitons, and the breather bends towards to the first-order rogue waves of Eqs. (1.2), cf. Figs. 3(c,f).

Analogously, the second-order rogue waves - i.e. if $N=2$, have the form

$$
\left(\begin{array}{c}
u_{[r 2]} \\
v_{[r 2]}
\end{array}\right)=\left(\begin{array}{c}
u_{[r 1]} \\
v_{[r 1]}
\end{array}\right)+\frac{2\left(\lambda_{1}^{*}-\lambda_{1}\right) \phi_{1[1]}^{*}}{\left|\phi_{1[1]}\right|^{2}+\left|\phi_{2[1]}\right|^{2}+\left|\phi_{3[1]}\right|^{2}} \times\left(\begin{array}{c}
\phi_{2[1]} \\
\phi_{3[1]}
\end{array}\right),
$$

where

$$
\begin{aligned}
& \left(\begin{array}{l}
\phi_{1[1]} \\
\phi_{2[1]} \\
\phi_{3[1]}
\end{array}\right)=\mho_{1}=T_{1} \Omega_{1}+i \theta \Omega_{0}, \quad T_{1}=2 i \theta\left(I_{3}-P_{1}\right), \quad P_{1}=\frac{\mho_{0} \mho_{0}^{\dagger}}{\mho_{0}^{\dagger} \mho_{0}}, \\
& \Omega_{1}=\exp \left(\frac{\theta^{2} i t}{2}\right) A\left(F_{0} G_{1}+F_{1} G_{0}\right) Z_{0}+F_{0} G_{0} Z_{1}, \quad Z_{0}=\left(\begin{array}{c}
z_{1[0]} \\
z_{2[0]} \\
z_{3[0]}
\end{array}\right), \quad Z_{1}=\left(\begin{array}{l}
z_{1[1]} \\
z_{2[1]} \\
z_{3[1]}
\end{array}\right), \\
& F_{1}=\left(\begin{array}{ccc}
\frac{x}{3}\left(-\theta^{3} x^{2}+3 \theta^{2} x-3 \theta\right) & -\frac{i a_{1} x^{3}}{\theta^{2}} & \frac{i a_{2} x^{3}}{\theta^{2}} \\
\frac{i a_{1} x^{3}}{\theta^{2}} & f_{1}[22] & f_{1}[23] \\
\frac{i a_{2} x^{3}}{\theta^{2}} & f_{1}[32] & f_{1}[33]
\end{array}\right), \\
& G_{1}=\left(\begin{array}{ccc}
-\frac{t}{3}\left(-\theta^{6} t^{2}+3 \theta^{4} t+6 i \theta^{2}\right) & \frac{a_{1} t}{3 \theta}\left(-\theta^{6} t^{2}+3 \theta^{2}\right) & \frac{-a_{2} t}{3 \theta}\left(-\theta^{6} t^{2}+3 \theta^{2}\right) \\
\frac{-a_{1} t}{3 \theta}\left(-\theta^{6} t^{2}+3 \theta^{2}\right) & g_{1}[22] & g_{1}[23] \\
\frac{-a_{2} t}{3 \theta}\left(-\theta^{6} t^{2}+3 \theta^{2}\right) & g_{1}[32] & g_{1}[33]
\end{array}\right)
\end{aligned}
$$

with

$$
\begin{aligned}
& f_{1}[22]=\frac{x}{3 \theta^{2}}\left(-\theta^{3} a_{1}^{2} x^{2}-3 a_{1}^{2} \theta^{2} x+3 \theta \exp (x \theta) a_{2}^{2}-3 \theta a_{1}^{2}\right), \\
& f_{1}[23]=\frac{-a_{1} a_{2} x}{3 \theta^{2}}\left(-\theta^{3} x^{2}-3 x \theta^{2}+3 \theta \exp (x \theta)-3 \theta\right), \\
& f_{1}[32]=\frac{a_{1} a_{2} x}{3 \theta^{2}}\left(-\theta^{3} x^{2}-3 x \theta^{2}+3 \theta \exp (x \theta)-3 \theta\right), \\
& f_{1}[33]=\frac{-x}{3 \theta^{2}}\left(-\theta^{3} a_{2}^{2} x^{2}-3 a_{2}^{2} \theta^{2} x+3 \theta \exp (x \theta) a_{1}^{2}-3 \theta a_{2}^{2}\right), \\
& g_{1}[22]=\frac{t}{3}\left(i a_{1}^{2} \theta^{4} t^{2}+3 a_{1}^{2} \theta^{2} t-6 i a_{1}^{2}+6 i a_{2}^{2} \exp \left(\frac{i t}{2} \theta^{2}\right)\right), \\
& g_{1}[23]=\frac{-a_{1} a_{2} t}{3}\left(i \theta^{4} t^{2}+3 \theta^{2} t-6 i+6 i \exp \left(\frac{i t}{2} \theta^{2}\right)\right), \\
& g_{1}[32]=\frac{-a_{1} a_{2} t}{3}\left(i \theta^{4} t^{2}+3 \theta^{2} t-6 i+6 i \exp \left(\frac{i t}{2} \theta^{2}\right)\right), \\
& g_{1}[33]=\frac{t}{3}\left(i a_{2}^{2} \theta^{4} t^{2}+3 a_{2}^{2} \theta^{2} t-6 i a_{2}^{2}+6 i a_{1}^{2} \exp \left(\frac{i t}{2} \theta^{2}\right)\right) .
\end{aligned}
$$




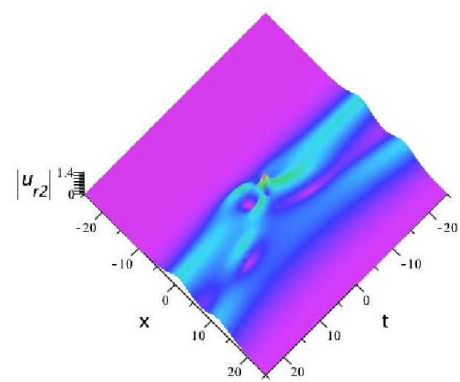

a)

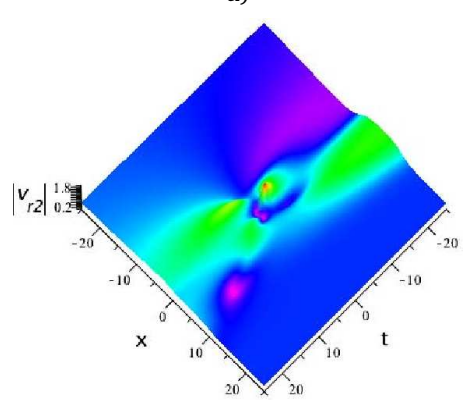

d)

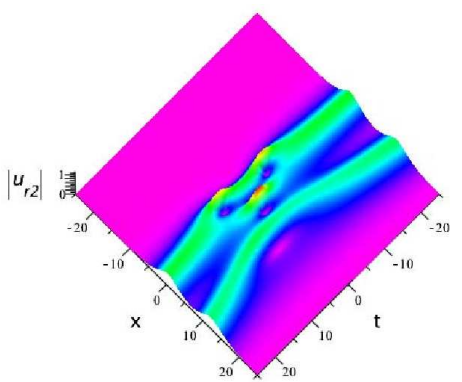

b)

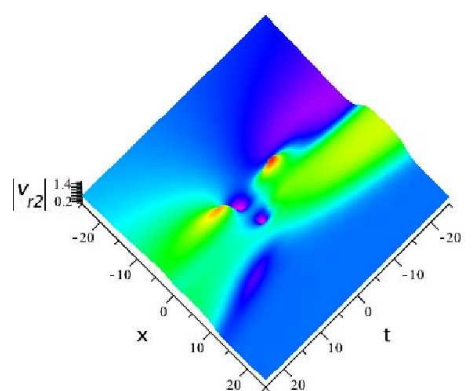

e)

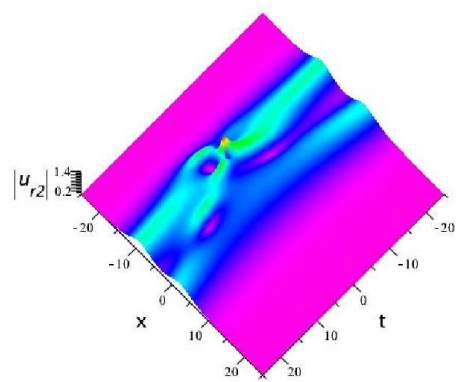

c)

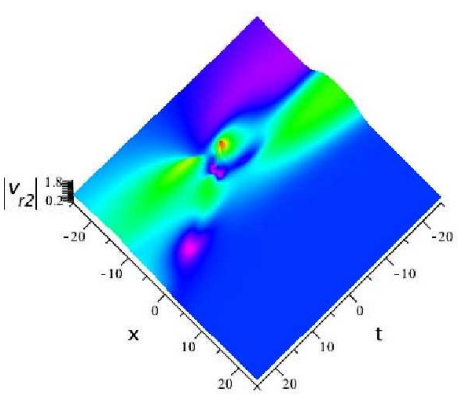

f)

Figure 4: Second-order rogue waves from Eqs. (4.2), $a_{1}=0, a_{2}=1 / 2, k=0, r_{0}=r_{1}=0, l_{1}=l_{2}=l_{3}=1$. $(a, d) s_{0}=s_{1}=0,(b, e) s_{0}=0, s_{1}=10,(c, f) s_{0}=10, s_{1}=0$.

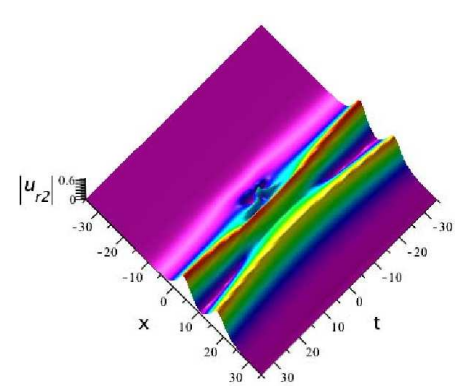

a)

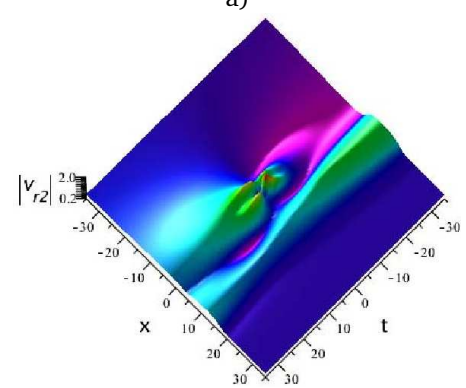

d)

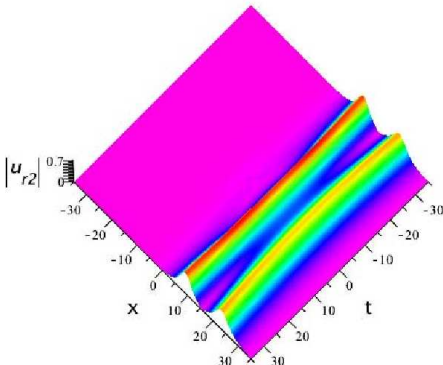

b)

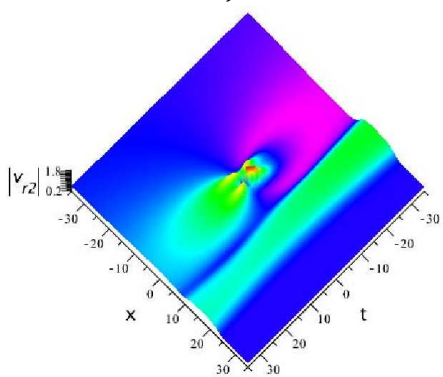

e)

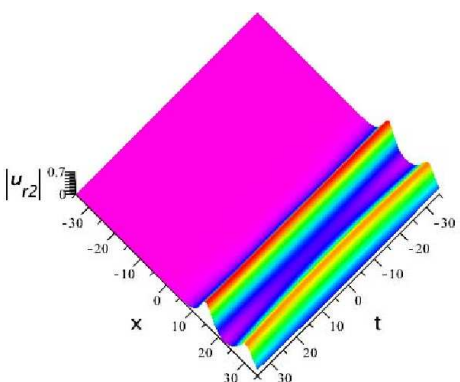

c)

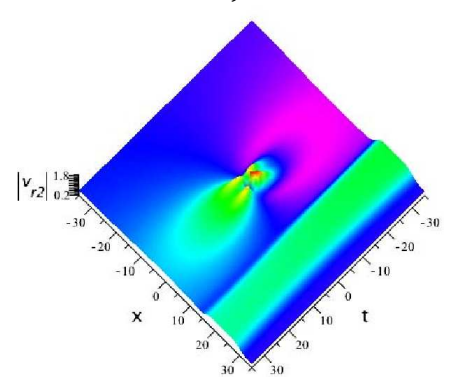

f)

Figure 5: Second-order rogue waves obtained from Eqs. (4.2), $a_{1}=0, a_{2}=1 / 2, k=0, r_{0}=r_{1}=s_{0}=s_{1}=$ $0, l_{1}=l_{2}=1$. $(a, d) l_{3}=5,(b, e) l_{3}=50,(c, f) l_{3}=1000$. 
Fig. 4 shows that the shape of the second-order rogue wave of $u_{r 2}$ changes when $s_{1}$ grows. The solution contains a single peak and splits into a wave with three peaks. The solution is called the three sisters or the rogue wave triplet. It can be noted that the change of $s_{2}$ has no effect on $u_{r 2}$ but only on $v_{r 2}$. The top line in Fig. 5 representing $u_{r 2}$, displays the interaction between second-order rogue waves and bright-soliton waves, whereas the next line demonstrates the interaction between second-order rogue waves and dark-soliton waves of $v_{r 2}$.

The $N$-order rogue waves can be constructed by the same method, However, the corresponding calculations are tedious, the expressions cumbersome, and are omitted here.

\section{Conclusions and Discussions}

We study the breather and rogue waves related to the Eqs. (1.2) via the Darbouxdressing transformation. Based on the Lax pair, suitable periodic seed solution and the Taylor series expansion, the novel solutions (novel breather solution and novel rogue waves) are constructed. $a_{1}, a_{2}, k, \lambda, l_{i}, r_{i}, s_{i}, i=1,2,3$ are all free parameters, which play an important role in controlling the dynamic properties. Visualisation of these solutions can help to further understand the characteristics of the Eqs. (1.2). We also hope that in the future, such solutions can be observed in experiments.

\section{Acknowledgements}

The authors express sincere thanks to the editors and reviewers for their valuable comments.

This work is supported by the National Natural Science Foundation of China (Nos. 71690242, 11731014, 12001241), by the Basic Research Program of Jiangsu Province (No. BK20200885), by the Graduate Research and Practice Innovation Program of Jiangsu Province (No. 1812000024432) and by the Young Science and Technology Talents Promotion Project for Zhenjiang Science and Technology Association.

\section{References}

[1] N. Akhmediev, A. Ankiewicz and J. Soto-Crespo, Rogue waves and rational solutions of the nonlinear Schrödinger equation, Phys. Rev. E 80, 026601 (2009) .

[2] N. Akhmediev, A. Ankiewicz and M. Taki, Waves that appear from nowhere and disappear without a trace, Phys. Lett. A 373, 675-678 (2009).

[3] F. Baronio, A. Degasperis, M. Conforti and S. Wabnitz, Solutions of the vector nonlinear Schrödinger equations: evidence for deterministic rogue waves, Phys. Rev. Lett. 109, 044102 (2012).

[4] T.B. Benjamin and J.E. Feir, The disintegration of wave trains on deep water, J. Fluid. Mech. 27, 417-430 (1976).

[5] Y.V. Bludov, V.V. Konotop and N. Akhmediev, Vector rogue waves in binary mixtures of BoseEinstein condensates, Eur. Phys. J. Spec. Top. 185, 169-180 (2010). 
[6] J.B. Chen and D.E. Pelinovsky, Periodic travelling waves of the modified KdV equation and rogue waves on the periodic background, J. Nonlinear. Sci. 29, 2797-2843 (2019).

[7] J.B. Chen, D.E. Pelinovsky, R.E. White, Periodic standing waves in the focusing nonlinear Schrödinger equation: Rogue waves and modulation instability, Phys. D 405, 132378 (2020).

[8] B.F. Feng, L.M. Ling and D.A. Takahashi, Multi-breather and high-order rogue waves for the nonlinear Schrödinger equation on the elliptic function background, Stud. Appl. Math. 144 (1), 46-101 (2020).

[9] B.L. Guo and L.M. Ling, Rogue wave, breathers and bright-dark-rogue solutions for the coupled Schrödinger equations, Chinese. Phys. Lett. 28, 110202-110202 (2011).

[10] D. Guo, S.F. Tian, X.B. Wang and T.T. Zhang, Dynamics of lump solutions, rogue wave solutions and traveling wave solutions for a $(3+1)$-dimensional VC-BKP equation, East Asian J. Appl. Math. 9, 780-796 (2019).

[11] T. Kanna, M. Lakshmanan, P.T. Dinda and N. Akhmediev, Soliton collisions with shape change by intensity redistribution in mixed coupled nonlinear Schrödinger equations, Phys. Rev. E 73(2), 026604 (2006).

[12] D. Kristian, E.K. Harald and M. Peter, On oceanic rogue waves, Annu Rev Fluid Mech 40(1), 287-310 (2008).

[13] M. Li, H. Liang, T. Xu and C.J. Liu, Vector rogue waves in the mixed coupled nonlinear Schrödinger equations, Eur. Phys. J. Plus. 131(4) (2016).

[14] L.M. Ling, L. Zhao and B.L. Guo, Darboux transformation and classification of solution for mixed coupled nonlinear Schrödinger equations, Commun. Nonlinear. Sci. Numer. Simul. 32, 285-304 (2016).

[15] Y. Ma, The perturbed plane-wave solutions of the cubic Schrödinger equation, Stud. Appl. Math. 60, 43-58 (1979).

[16] W.M. Moslem, P. Shukla and B. Eliasson, Surface plasma rogue waves, EPL. 96, 25002 (2011).

[17] G. Mu and Z.Y. Qin, Nth-Order rogue waves to nonlinear Schrödinger equation revisited: A variable separation technique, J. Phys. Soc. Jan. 83,104001 (2014) .

[18] G. Mu, Z.Y. Qin and R. Grimshaw, Dynamics of rogue waves on a multisoliton background in a vector nonlinear Schrödinger equation, SIAM J. Appl. Math. 75, 1-20 (2015) .

[19] D.H. Peregrine, Water waves, nonlinear Schrödinger equations and their solutions, J. Australian. Math. Soc. Ser. B 25 (1983).

[20] D. Solli, C. Ropers, P. Koonath and B. Jalali, Optical rogue waves, Nature 450, 1054-1057 (2007).

[21] S.F. Tian, The mixed coupled nonlinear Schrödinger equation on the half-line via the Fokas method, Proc. Math. Phys. Eng. Sci. 472, 20160588 (2016).

[22] M. Vijayajayanthi, T. Kanna and M. Lakshmanan, Bright-dark solitons and their collisions in mixed N-coupled nonlinear Schrödinger equations, Phys. Rev. A 77, 013820 (2008).

[23] X.B. Wang and B. Han, Characteristics of rogue waves on a soliton background in a coupled nonlinear Schrödinger equation, Math. Method. Appl. Sci. 42, 2586-2596 (2019).

[24] X.B. Wang and B. Han, The three-component coupled nonlinear Schrödinger equation: Rogue waves on a multi-soliton background and dynamics, EPL 126, 15001 (2019).

[25] X.B.Wang and B. Han, The Kundu-nonlinear Schrödinger equation: Breathers, rogue waves and their dynamics, J Phys Soc Jan. 89, 014001 (2020).

[26] X.W. Yan, Lax pair, Darboux-dressing transformation and localized waves of the coupled mixed derivative nonlinear Schrödinger equation in a birefringent optical fiber, Appl. Math. Lett. 107, 106414 (2020).

[27] X.W. Yan, S.F. Tian, M.J. Dong and T.T. Zhang, Rogue waves and their dynamics on bright-dark soliton background of the coupled higher order nonlinear Schrödinger equation, J. Phys. Soc. 
Jan. 88, 074004 (2019).

[28] X.W. Yan and J.F. Zhang, Coupled cubic-quintic nonlinear Schrödinger equation: novel brightdark rogue waves and dynamics, Nonlinear. Dynam. 100, 3733-3743 (2020).

[29] Z.Y. Yan, Financial rogue waves, Commun. Theor. Phys. 54, 947-949 (2010).

[30] Z.Y. Yan, Vector financial rogue waves, Fuel \& Energy Abstracts 375(48), 4274-4279 (2011).

[31] R. Ye, Y. Zhang, Q. Zhang and X. Chen, Vector rational and semi-rational rogue wave solutions in the coupled complex modified Korteweg-de Vries equations, Wave Motion 92, 102425 (2020).

[32] Y.Q. Yuan, B. Tian, H. Chai, X.Y. Wu and Z. Du, Vector semirational rogue waves for a coupled nonlinear Schrödinger system in a birefringent fiber, Appl. Math. Lett. 87, 50-56 (2019).

[33] G.Q. Zhang, Z.Y. Yan, X.Y. Wen and Y. Chen, Interactions of localized wave structures and dynamics in the defocusing coupled nonlinear Schrödinger equations, Phys. Rev. E 95 (4), 42201 (2017).

[34] L.D. Zhang, S.F. Tian, W.Q. Peng, T.T. Zhang and X.J. Yan, The dynamics of lump, lumpoff and rogue wave solutions of $(2+1)$-dimensional Hirota-Satsuma-Ito equations, East Asian J. Appl. Math. 10, 243-255 (2020).

[35] X.M. Zhou, S.F. Tian, J.J. Yang and J.J. Mao, The Riemann-Hilbert approach and N-soliton solutions of a four-component nonlinear Schrödinger equation, East Asian J. Appl. Math. 11, 143-163 (2021). 